\title{
Water wave experiments Tourbillonde
}

A. Maurel

Institut Langevin - Paris

V. Pagneux

LAUM - Le Mans

P. Petitjeans

Students:

$\mathrm{PMMH}$ - Paris

P. Cobelli, A. Prazda,

M. Chekroun, G. Lagubeau 


\section{Water wave experiments Tourbillonde}

A. Maurel

Institut Langevin - Paris

V. Pagneux

LAUM - Le Mans

P. Petitjeans

PMMH - Paris

Students:

P. Cobelli, A. Prazda,

M. Chekroun, G. Lagubeau






\section{Water wave experiments Motivation}
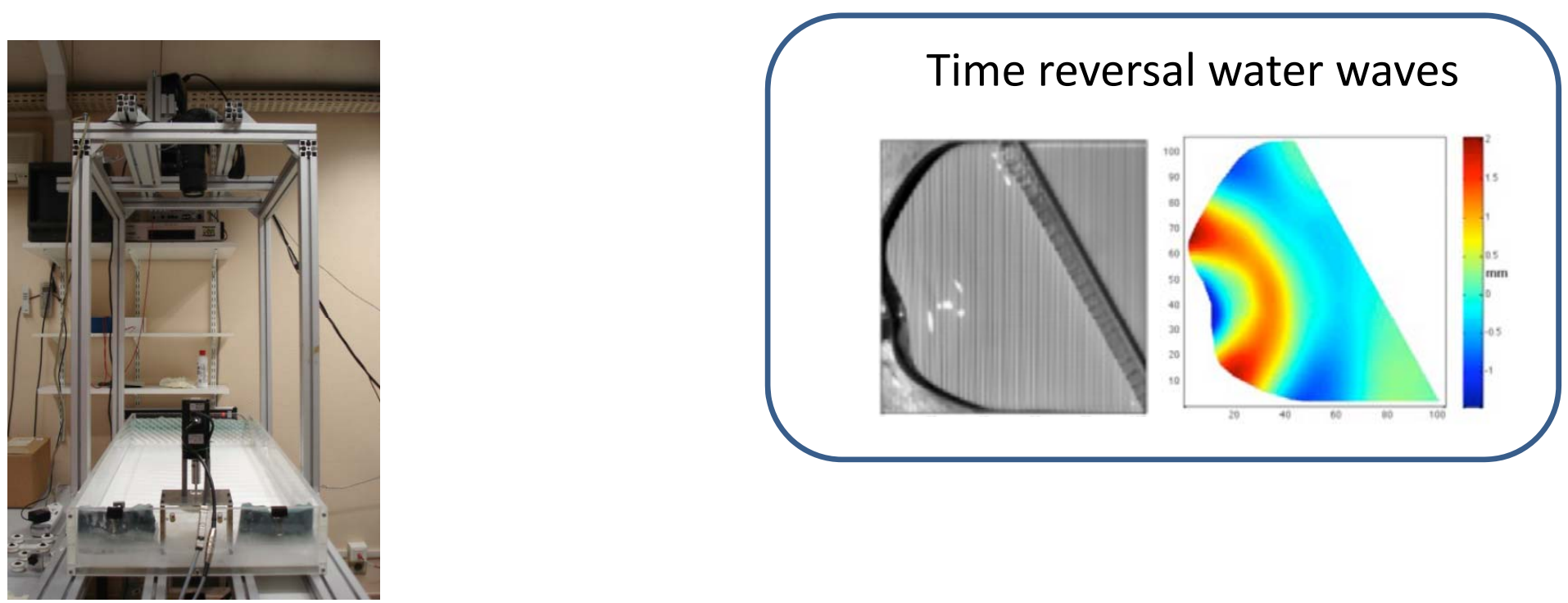

Wave emission by a vortex
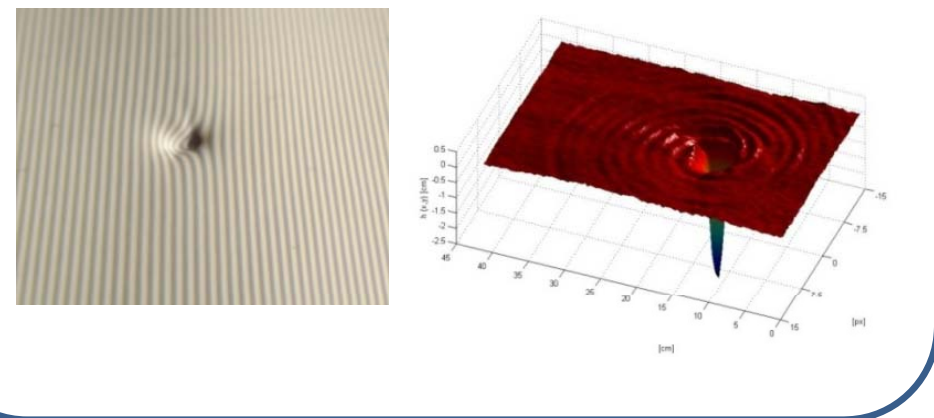

Interaction wave - vortex

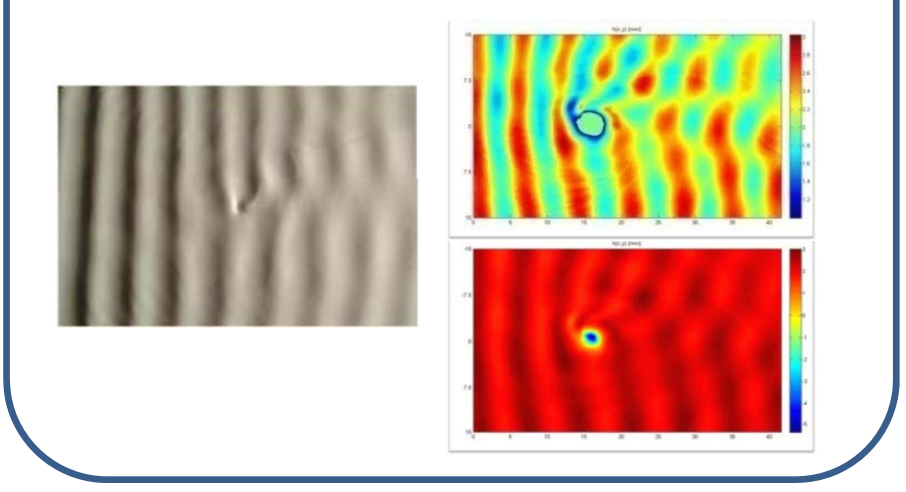




\section{Water wave experiments}

\section{How to get quantitative measurements ?}

(instantaneous, global): Fourier Transform Profilometry






\section{Current subjects}

- Directional water wave emission by embedded sources

M. Chekroun

- Inverse measurement of underwater movement of the sea floor P. Cobelli

- Wave Turbulence served up on a plate

P. Cobelli, Coll. N. Mordant, ENS - Paris

- Azimuthal instability of drop impact

G. Labugeau,

Coll. C. Josserand, Institut Jean le rond d'Alembert, Univ. Paris 6

M. Fontelos, Dto Matemáticas, F. Ciencias, Univ. Autónoma de Madrid 
Directional water wave emission by embedded sources M. Chekroun

Water waves propagating through a square array of surface piercing cylinders

$$
\begin{gathered}
\left(\nabla_{2}^{2}+k^{2}\right) \phi=0, \\
\partial \phi / \partial \vec{n}=0,
\end{gathered}
$$
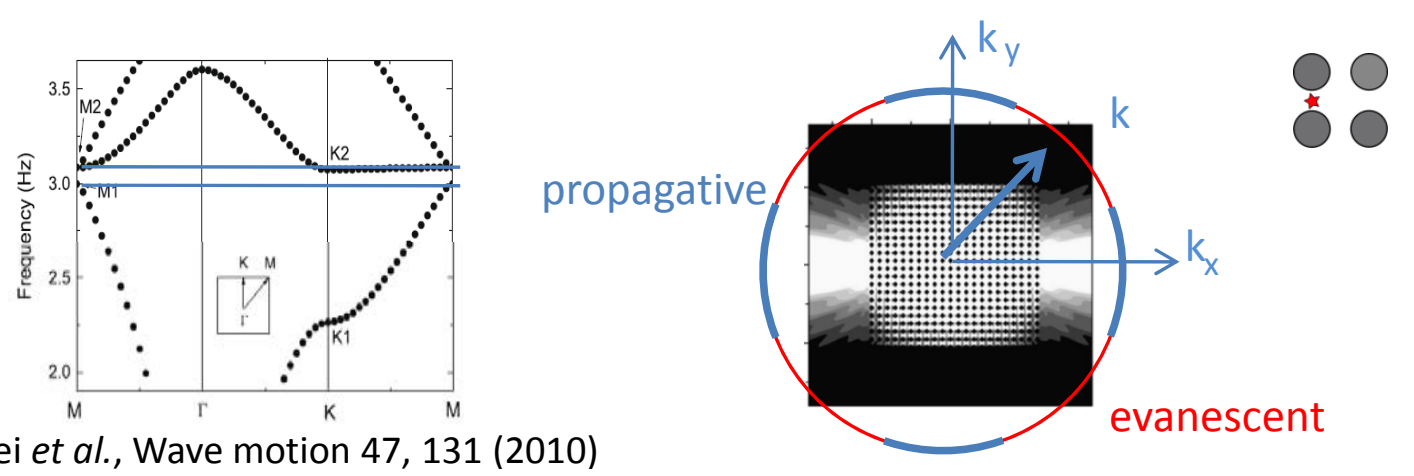

\section{Experiments}
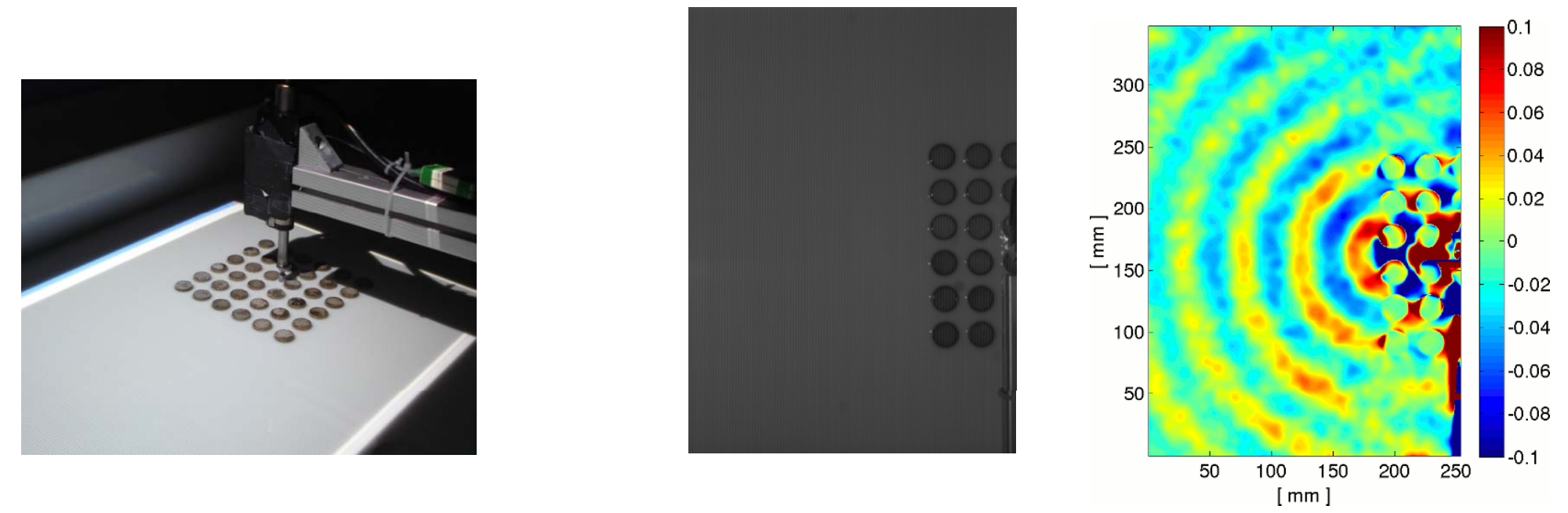
Experimental measurements ( $6 \times 6$ square array)

(at the working frequency)

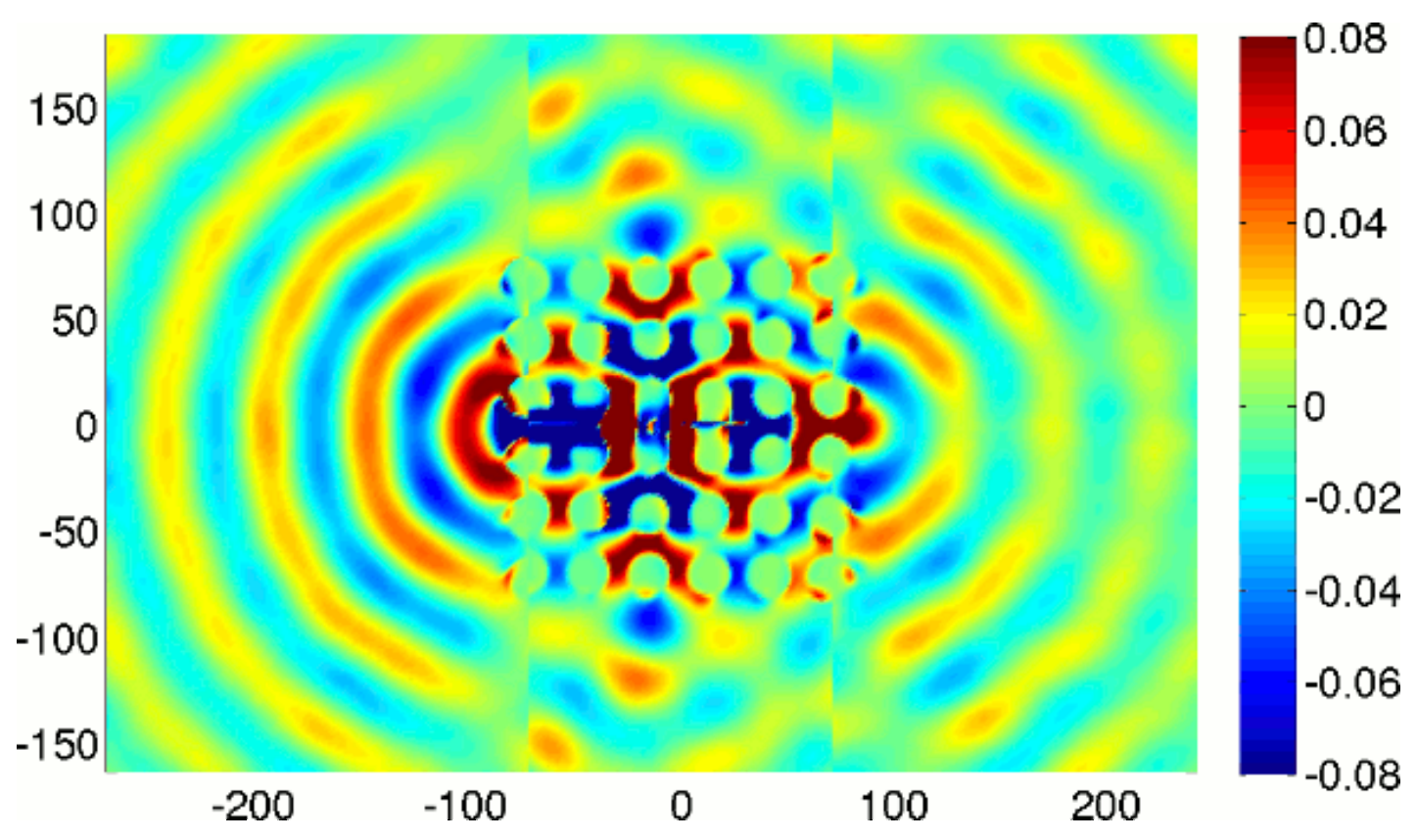


Experimental measurements (amplitude)

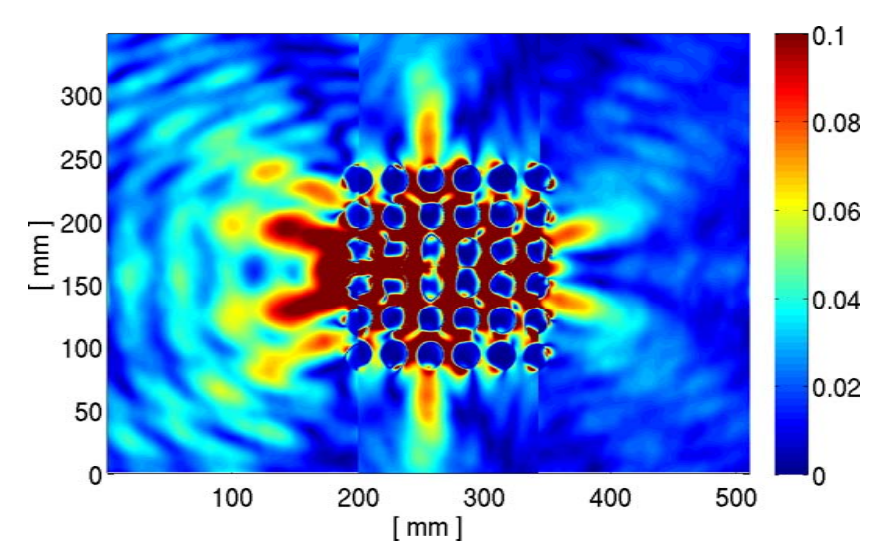

Numerics (with artificial attenuation)

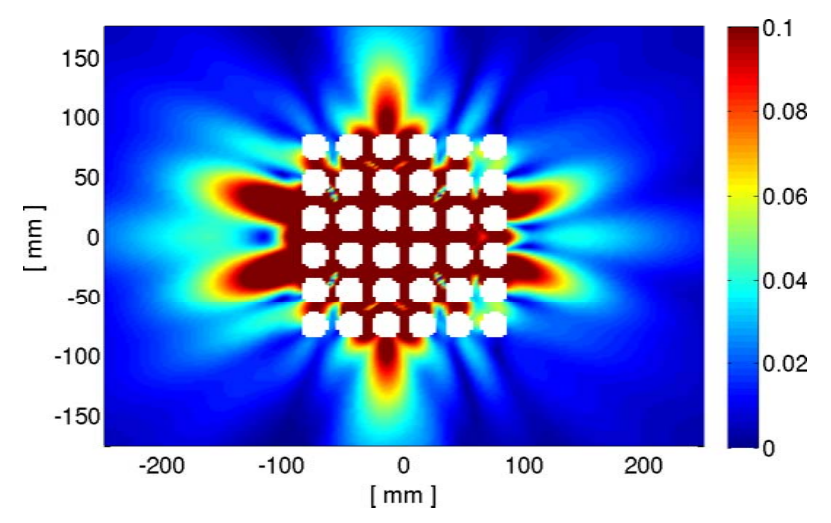

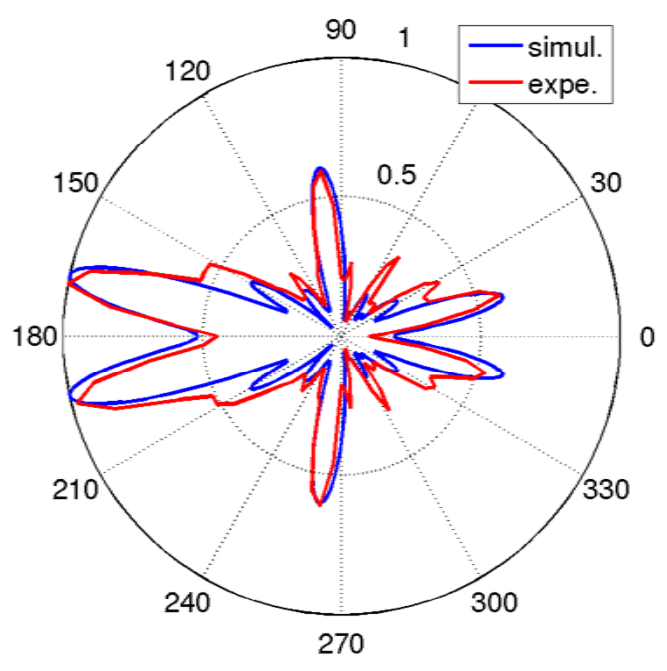


Inverse measurement of underwater movement of the sea floor P. Cobelli
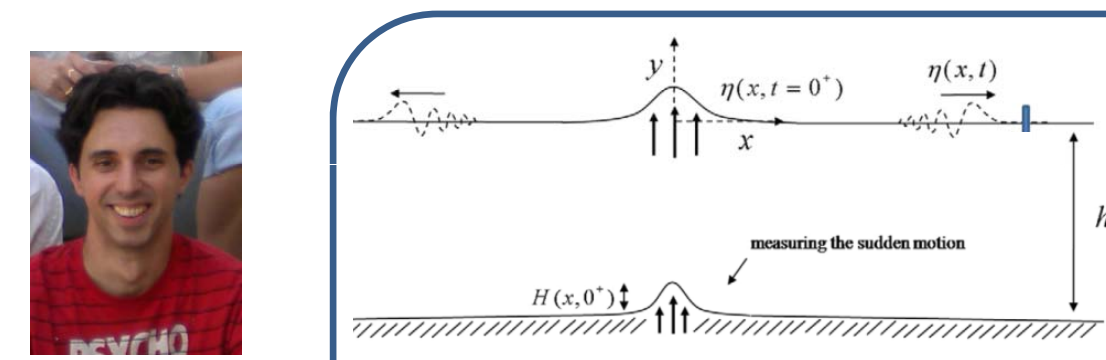

$\varphi_{x x}+\varphi_{y y}=0$ in water,

$\eta_{t}=\varphi_{y} \quad$ on $y=0$,

$\varphi_{t}+g \eta=0$ on $y=0$

$\varphi_{y}=H_{t} \quad$ on $y=-h_{0}$.

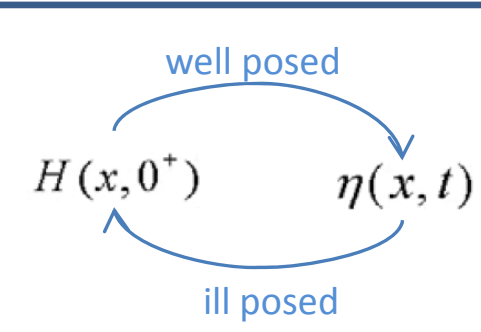

T.S. Jang et al./Wave Motion 47 (2010) 146-155

Regularization method (Landweber Fridman regularization scheme)

Experimental set up

-2D problem
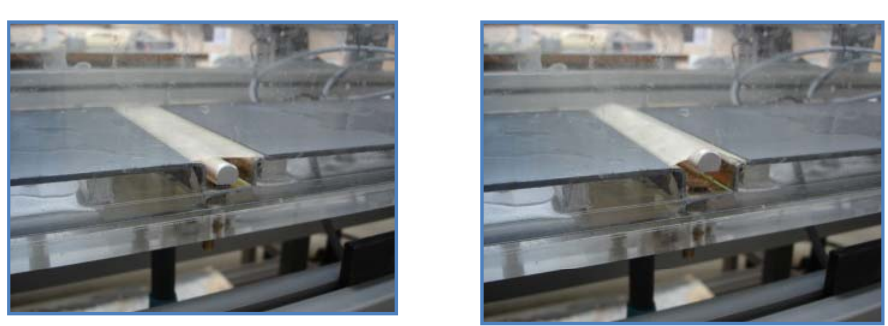

- axisymmetric 
Experimental measurements

Axisymmetric configuration
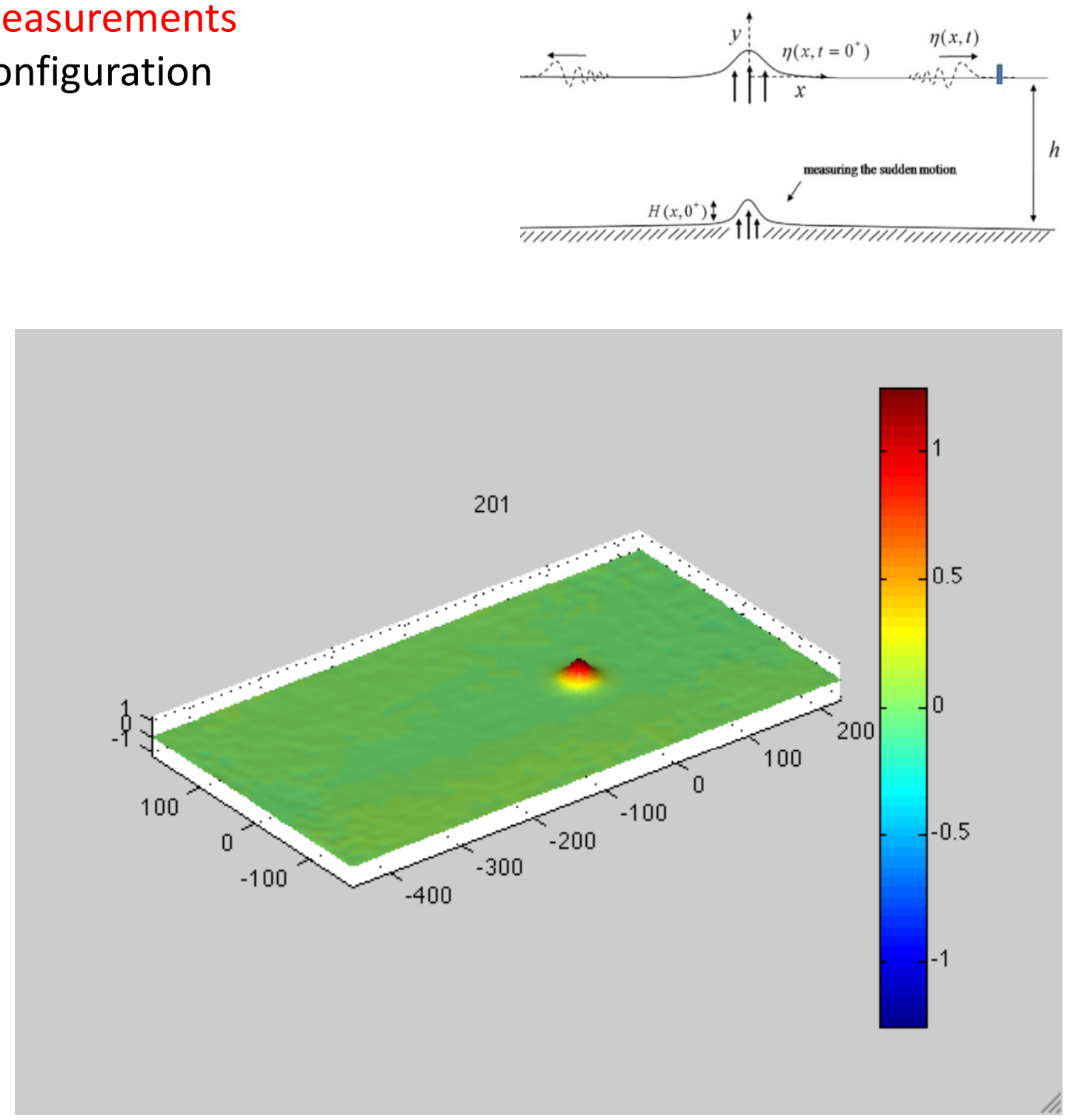
Result of the inversion

$$
\eta(x, t) \longrightarrow H\left(x, 0^{+}\right)
$$

Sea bottom motion $H\left(x, 0^{+}\right)$known (measured)

free surface displacement $\eta(x, t)$

Reconstructed bottom sea motion $H\left(x, 0^{+}\right)$
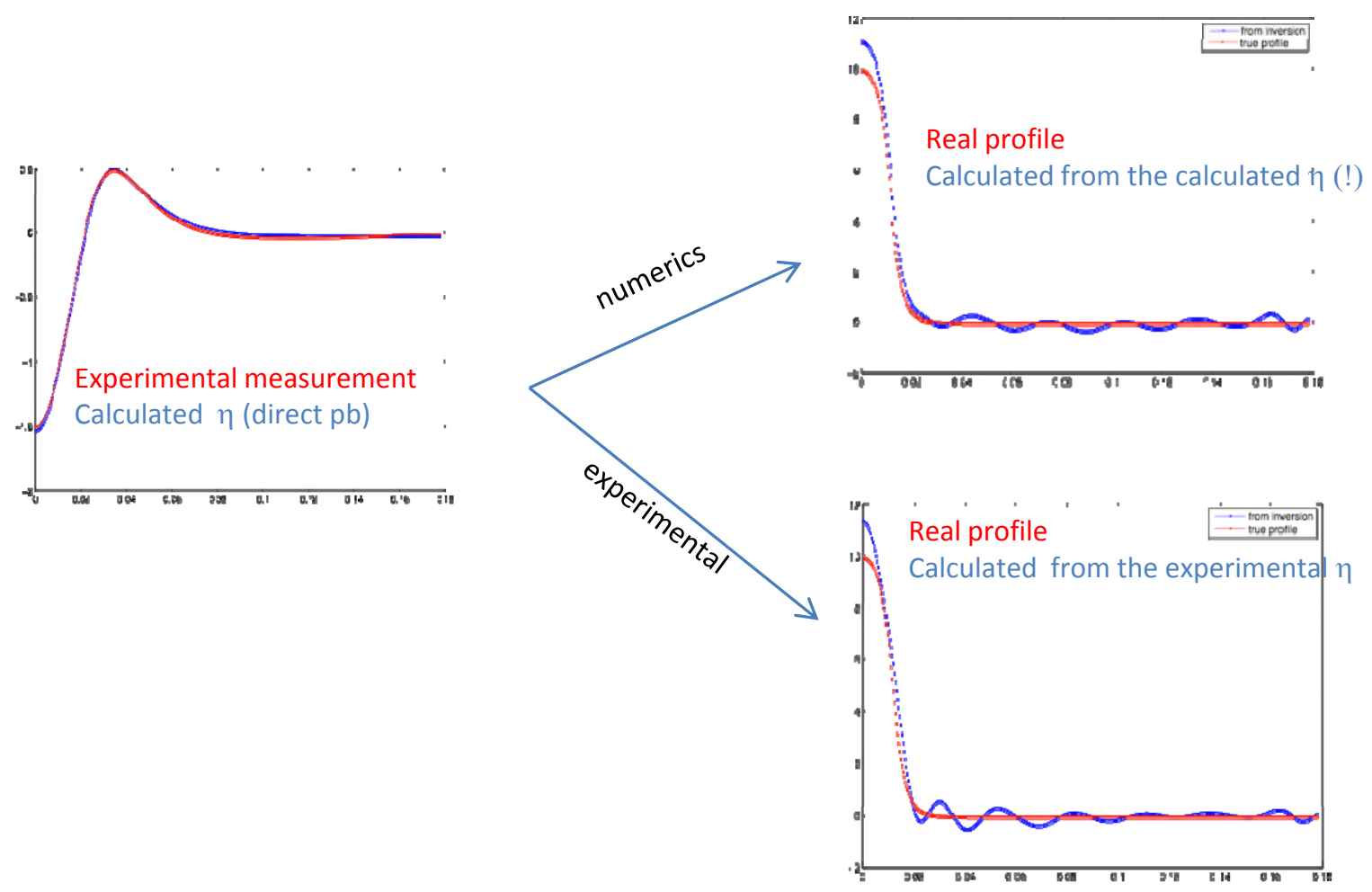


\section{Wave Turbulence served up on a plate}

P. Cobelli, Coll. N. Mordant, ENS - Paris

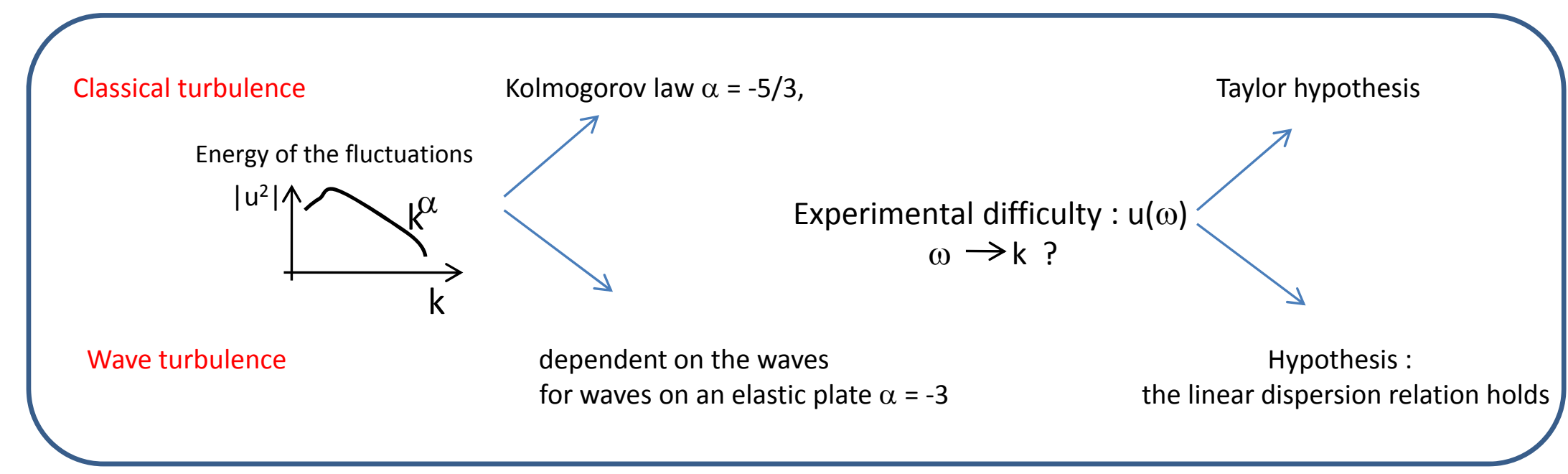

Experimental set up (N. Mordant ENS)
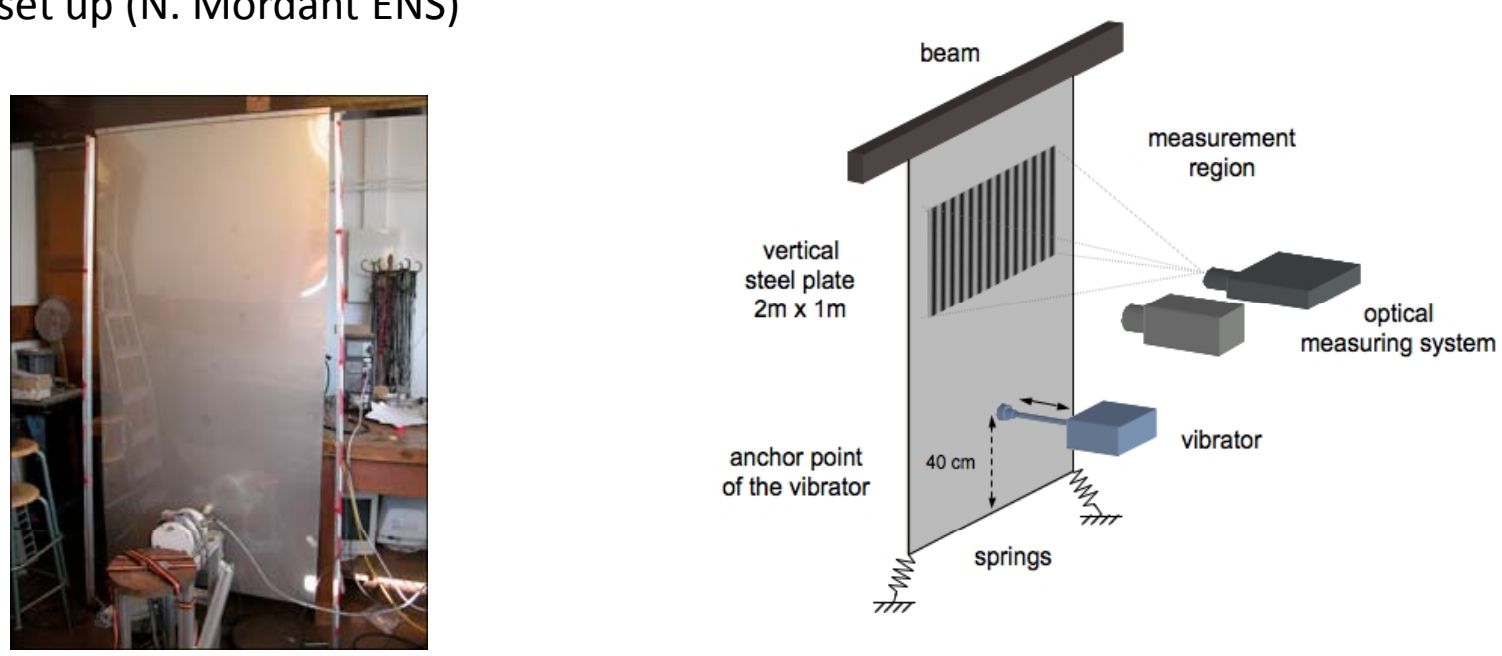
Fluctuation velocity $\mathrm{u}(\mathrm{x}, \mathrm{t})$

Experimental measurements
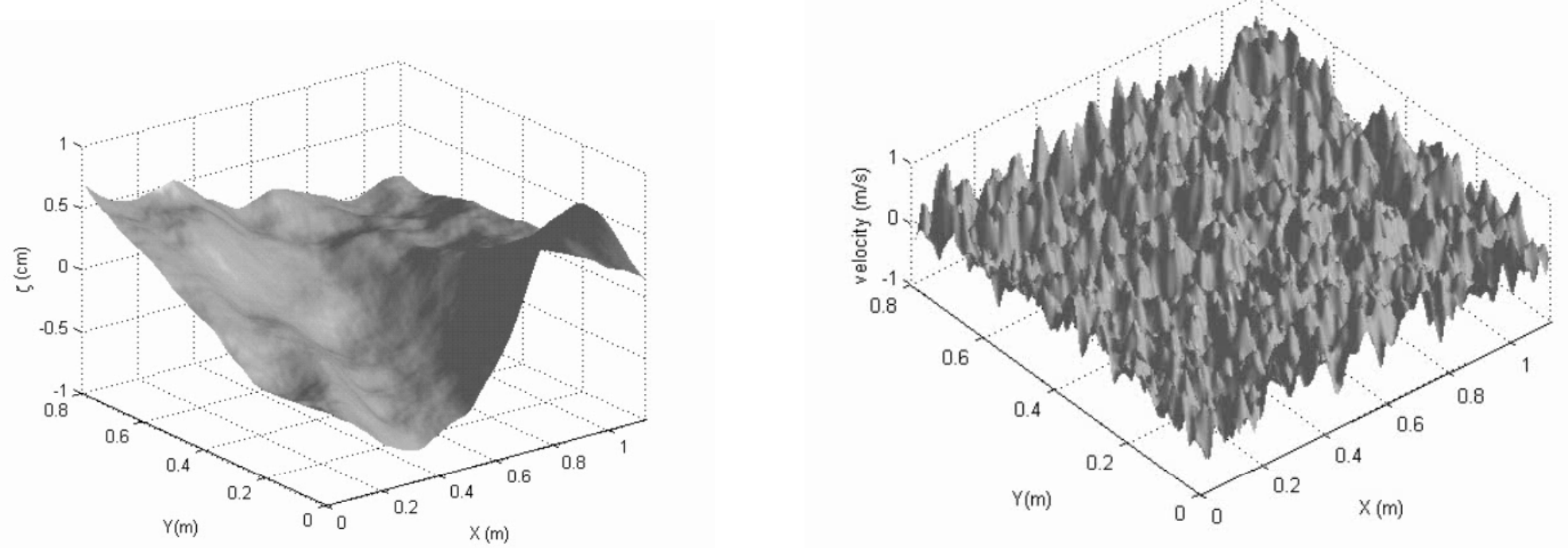

First experimental evidence of the dispersion relation in wave turbulence $\left|u^{2}\right|(k, \omega)$
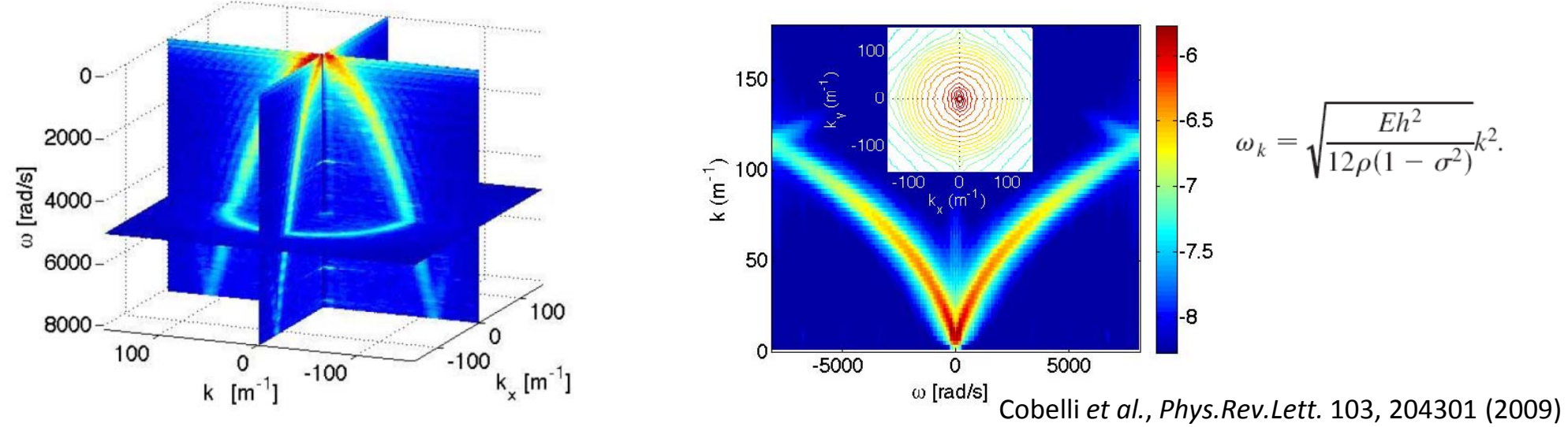
Azimuthal instability of drop impact

G. Lagubeau,

Coll. C. Josserand, Institut Jean le rond d'Alembert, Univ. Paris 6

M. Fontelos, Dto Matemáticas, F. Ciencias, Univ. Autónoma de Madrid
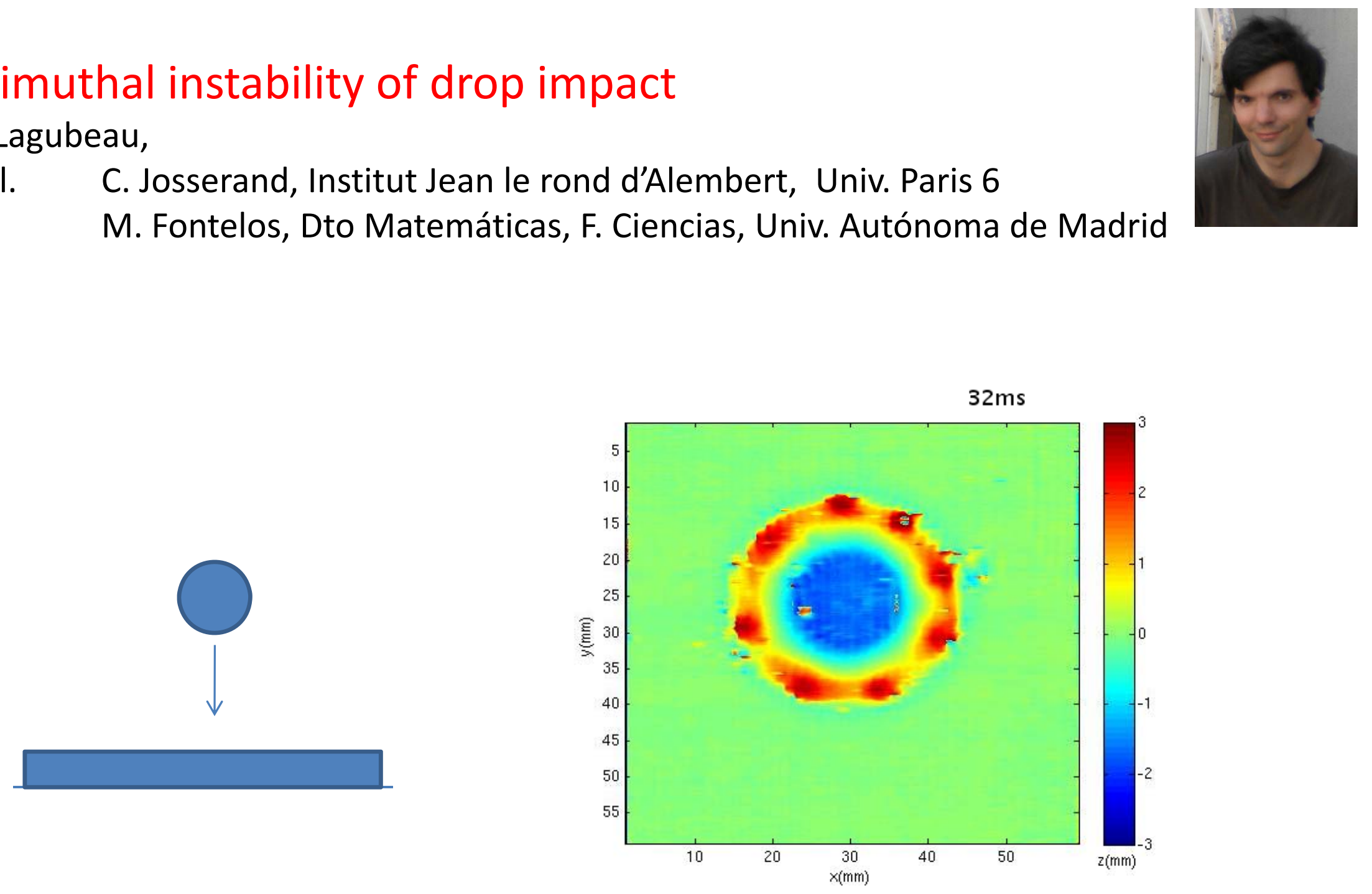